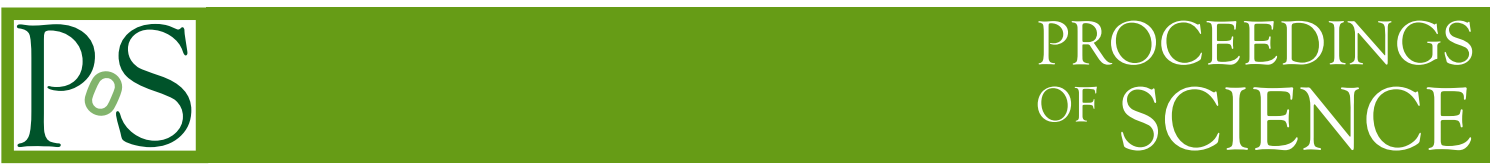

\title{
Model Unspecific Search in CMS (MUSiC)
}

\author{
Lorenzo Vigilante on behalf of the CMS Collaboration. ${ }^{a, 1, *}$ \\ ${ }^{a}$ RWTH Aachen University, \\ Otto-Blumenthal-Straße 16, Aachen, Germany \\ E-mail: lorenzo.vigilante@cern.ch
}

\begin{abstract}
The Model Unspecific Search in CMS analysis searches for anomalies in data that can be probed for new physics phenomena based purely on the comparison of the recorded data to the expectation according to the standard model, obtained from simulations. Events selected with at least one lepton (electron or muon) are classified into several hundred event classes based on their final state topology, taking electrons, muons, photons, jets, b-tagged jets, and missing transverse momentum into account. An automated search algorithm subsequently surveys kinematic distributions of the data for deviations from the SM expectation without any explicit input of any particular new physics models. The search strategy and the results of the MUSiC analysis using $35.9 \mathrm{fb}^{-1}$ of data recorded by the CMS detector at the CERN LHC during proton-proton collisions at a center of mass energy of $13 \mathrm{TeV}$ are presented here.
\end{abstract}

40th International Conference on High Energy physics - ICHEP2020

July 28 - August 6, 2020

Prague, Czech Republic (virtual meeting)

${ }^{*}$ Speaker 


\section{Introduction}

Fundamental interactions in particle physics are well described by the standard model (SM). However, several phenomena such as dark matter, neutrino oscillation, and the matter-antimatter asymmetry are still without a comprehensive explanation.

The Model Unspecific Search in CMS (MUSiC) is a general model-independent search for beyond SM phenomena that aims to identify discrepancies between a Monte Carlo (MC) simulation of SM processes in hundreds of different final states, and the observed data collected by the Compact Muon Solenoid (CMS) [1] at the CERN Large Hadron Collider.

This analysis complements dedicated search analyses by significantly expanding the range of final states covered, using the largest data set to date to probe phase space regions beyond the reach of previous general searches.

This article describes the MUSiC analysis [2] performed with the full proton-proton collisions dataset at $\sqrt{s}=13 \mathrm{TeV}$ collected by CMS during 2016, corresponding to an integrated luminosity of $35.9 \mathrm{fb}^{-1}$.

\section{Analysis strategy}

The MUSiC analysis selects events with at least one muon or electron, followed by the classification of data and $\mathrm{MC}$ events in different event classes based on their final state topology, taking electrons, muons, photons, jets, b-tagged jets, and missing transverse momentum into account. Three different types of event classes are defined: Exclusive event classes for events containing only those selected objects that are specified for the event class; Inclusive event classes contain events that include a nominal set of selected objects, but may contain additional objects; Jet-inclusive event classes are defined as inclusive classes but additional object are restricted to jets.

A scan for deviation between data and SM simulation is performed on three kinematic variables: the sum of the transverse momentum $\left(S_{T}\right)$ of all the physics objects in an event; the invariant mass $M$ or the transverse mass $M_{T}$ for classes with $p_{T}^{\text {miss }}$; the missing transverse momentum $p_{T}^{\text {miss }}$. A $p$-value has been defined in [2] to describe the agreement between simulation and data.

The $p$-values are evaluated in all the possible connected bin regions in a distribution. The region with the smallest $p$-value is defined as Region of Interest (RoI).

To provide a measure of finding a deviation of equal or lesser compatibility anywhere in the distribution, $p$-values can be converted into $\tilde{p}$-values correcting for the Look Elsewhere Effect.

\section{Results}

The MUSiC classification algorithm has defined 498 exclusive event classes and 571 (530) inclusive (jet-inclusive) event classes with at least one data event. For each event class we have performed scans based on the total yield of each event class. Figure 1 shows both the distribution for exclusive event classes that contain exactly two electrons and any number of jets or b-jets. No particular event class shows a large and significant deviation between data and SM simulation beyond the expectation.

Due to the large number of different event classes and kinematic distributions scanned, a global overview of the scan is required. To produce a global overview of all event classes, the $\tilde{p}$-values 


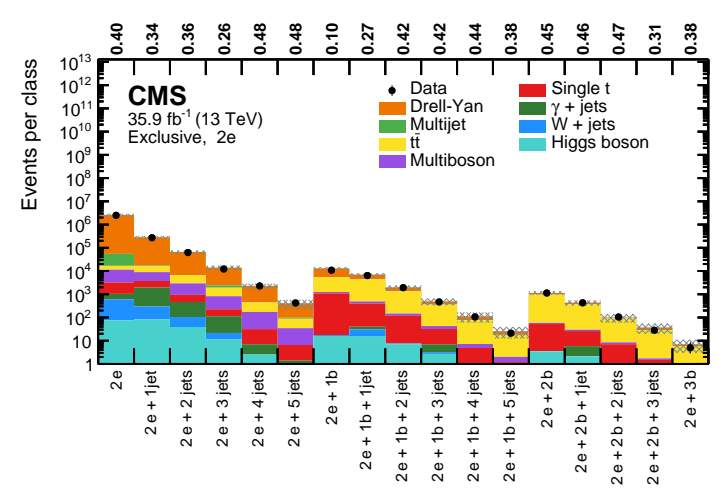

Figure 1: Overview of total event yields for exclusive event classes that contain exactly two electrons and any number of jets or b-jets. The values above the plot indicate the observed $p$-value for each event class [2].

evaluated for each kinematic distribution are summarized in a single histogram and compared with SM-only expectation obtained from pseudo-experiments. Figure 2 shows the global overview plots for the $M$ and $S_{T}$ RoI scans for the exclusive event classes.
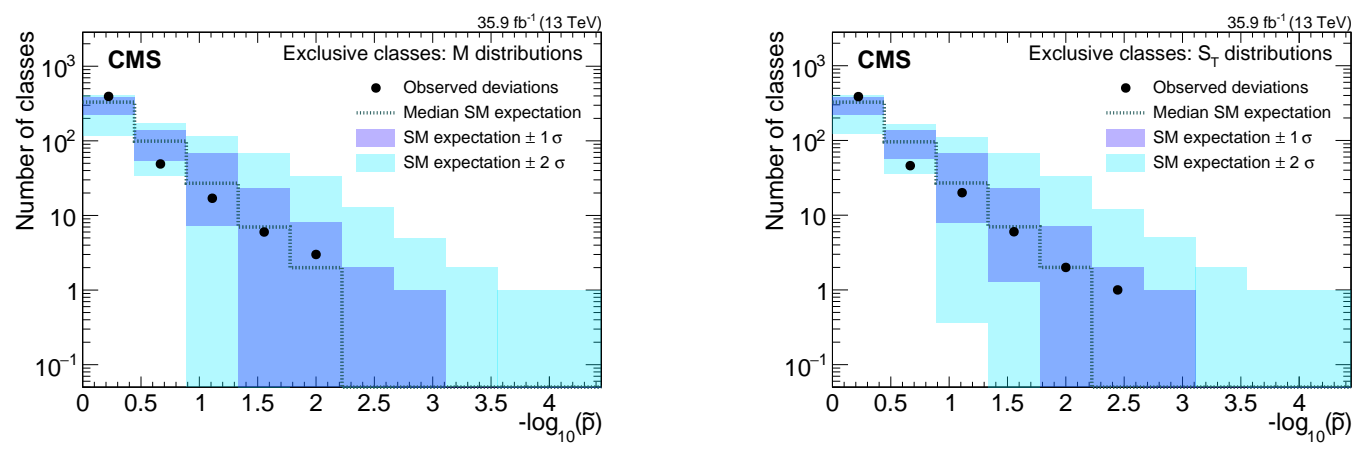

Figure 2: Overview of total event yields for event classes corresponding to the double-electron object groups (left) and the total event yield histogram for the top 20 exclusive event classes (right). The values above the plot indicate the observed $p$-value for each event class [2].

No significant deviations from the standard model expectations were found in the data analyzed by the MUSiC algorithm. A wide range of final-state topologies has been studied, and there is agreement between data and the standard model simulation given the experimental and theoretical uncertainties.

\section{References}

[1] CMS Collaboration, The CMS experiment at the CERN LHC, JINST 3 (2008) S08004.

[2] CMS Collaboration, MUSiC: a model unspecific search for new physics in protonproton collisions at $\sqrt{s}=13 \mathrm{TeV}$, CMS-EXO-19-008, CERN-EP-2020-171, CDS: https://cds.cern.ch/record/2740713. 